\title{
Persistent intracranial bleeding as a complication of hemispherectomy
}

\author{
D. R. OPPENHEIMER AND H. B. GRIFFITH
}

From the Departments of Neuropathology and Neurological Surgery, Radcliffe Infirmary, Oxford

Cerebral hemispherectomy was devised by Dandy (1928) as a radical treatment for glioma of the nondominant hemisphere. It has since been carried out sporadically for this condition, but long-term survivals have been understandably few. Krynauw (1950) applied the operation to cases of infantile hemiplegia with fits and behaviour disorders; and this form of treatment has been widely practised since then. In Oxford, between 1950 and 1961, hemispherectomy for infantile hemiplegia has been carried out on 17 patients. Of these 17 , four have subsequently died, one from an unknown cause. The other three have come to necropsy, and their brains have been examined in Oxford. All these survived for several years after operation, with an initial period of well-being followed by a period of slow deterioration ending in death. The clinical and anatomical findings in all these cases have been remarkably similar, and can be explained on the basis that repeated bouts of bleeding had occurred within the cranial cavity. At the time of death, all showed obstructive hydrocephalus, combined with

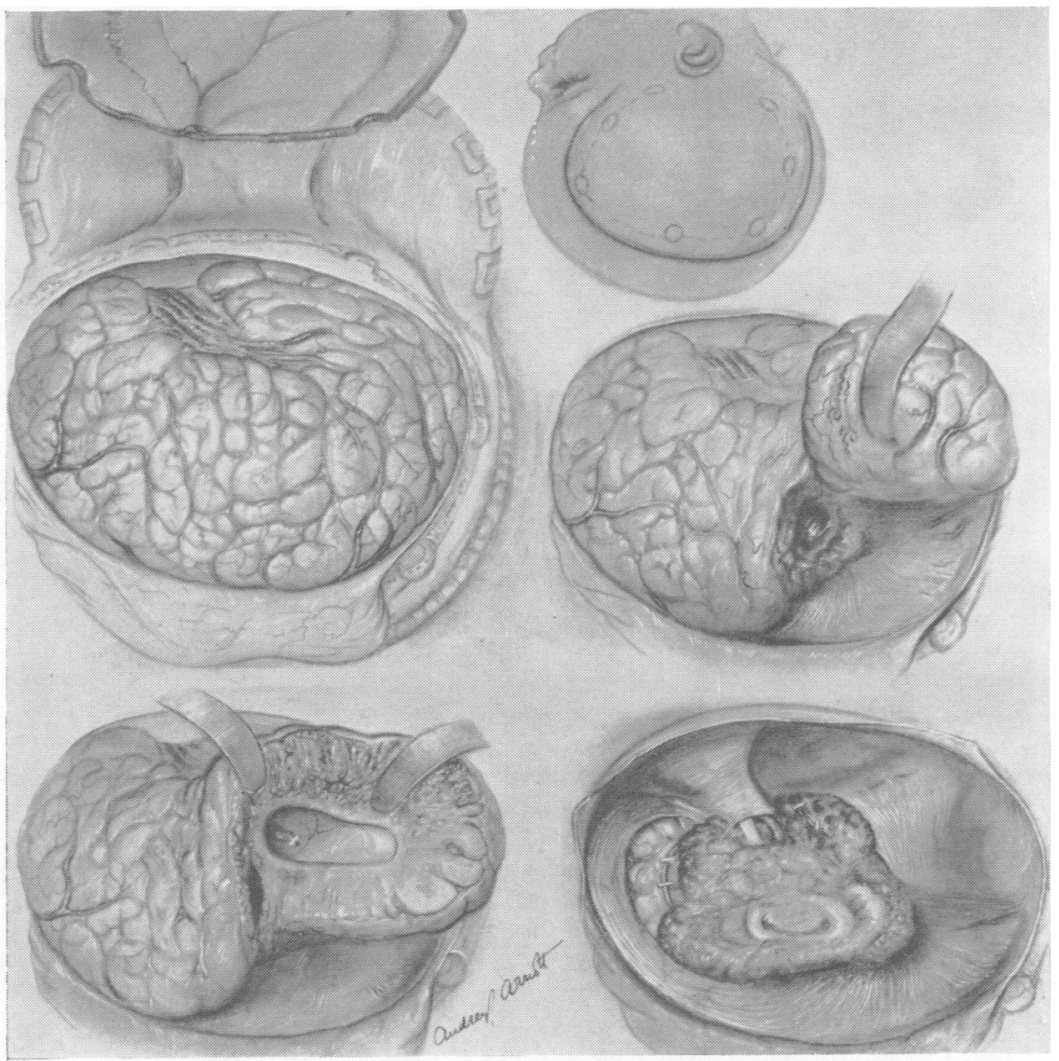

FIG. 1. The hemisphere is removed in three or four quadrants. 
superficial haemosiderosis of the central nervous system.

\section{OPERATIVE PROCEDURES}

A uniform one-stage technique was used, with a large lateral osteoplastic craniotomy (Fig. 1). The diseased hemisphere was removed in three or four blocks, leaving behind the gyrus rectus, hippocampus and hippocampal gyrus, part of the cingulate gyrus, thalamus, hypothalamus, and most of the basal nuclei. The choroid plexus was removed as far forward as the foramen of Monro. Haemostasis was by diathermy, with silver clips for the major vessels. No foreign material was left in the cavity. The cavity which remained was left in communication with the third and lateral ventricles via the foramen of Monro, and was closed without drainage after being filled with physiological saline solution. Lumbar punctures were usually done in the first post-operative days with the aim of promoting circulation of the spinal fluid. The operation is thus not strictly removal of the whole hemisphere but a complete callosotomy and an almost complete decortication, leaving the basal nuclei intact.

\section{CASE 1}

The birth, early milestones, and growth of this girl, L. du C., were normal until the age of 11 months, when she had a febrile illness ushered in by an adversive fit referable to the right cerebral hemisphere. She was opisthotonic for four days, drowsy for six days, and a left hemiplegia was apparent when she left hospital after one month. Her later development was slowed up, but she was able to walk at the age of 2 , and was speaking fairly well at 4 years. She was by this time subject to frequent temper tantrums. At age 5 she began to have frequent fits. These were minor, adversive, lasted a few seconds only and ended with a giggle or a shout. The first bout lasted for one month, then abated for over a year, but during this time her temper tantrums became more frequent and more severe. She had been attending an ordinary school, but had to be withdrawn from this because of a recurrence of fits at age $6 \frac{1}{2}, 18$ months after they had first appeared. The fits were now much more severe, lasted over an hour, again with tonic adversive posture and head banging at the breaking up of the fit. When seen at this stage, she was a fat child with left hemi-smallness, and a spastic left hemiparesis. Power was better preserved in the left leg than in the left arm, which was neglected. Her I.Q. at this time was 62 . An electroencephalogram (E.E.G.) showed a right temporal spike focus with a more widespread disturbance over the right hemisphere. The calvarium on the right was thick. Lumbar air encephalography showed right ventricular dilatation and a virtually normal left lateral ventricle. The spinal fluid was normal.

She was discharged on Tridione and Epanutin, which were ineffective, the fits becoming more severe and fre- 으 quent and the behaviour slightly more violent. She had a $Z$ right cerebral hemispherectomy in June 1954, when aged 8 years. The operation itself was uneventful.

The removed tissue weighed $140 \mathrm{~g}$. There was no gross scarring, but the meninges were thick. There was dense $\vec{\theta}$ subpial gliosis, and the cortex showed patchy cell loss, $T$ most marked in the frontal and parietal cortex, and elsewhere at the bases of sulci. There was myelin loss and $\stackrel{\text { ? }}{工}$ fibrous gliosis in the underlying white matter. No vascular lesions were seen.

Post-operatively, she ran a fever of $100^{\circ}$ to $101^{\circ} \mathrm{F}$. for three weeks. Lumbar punctures showed a clear xantho- $\stackrel{\vec{D}}{\stackrel{D}{ }}$ chromic fluid with the protein level gradually falling from 900 to $400 \mathrm{mg} . / 100 \mathrm{ml}$., and a pleocytosis in the 음 range of 100 w.b.c./c.mm. The deficit due to removal of $\frac{\overline{\bar{p}}}{\overrightarrow{2}}$ the hemisphere was a slight increase in the weakness of $\widehat{D}$ the left arm and a left homonymous hemianopia, but no more. Her behaviour was better. She was now able to $\tilde{\Phi}$ concentrate, and indeed did this for the first time. There $\overrightarrow{0}$ was a major convulsion on the 15 th post-operative day, but although she had a few further fits in the years follow- $\vec{\omega}$ ing, they were all of the minor type first described.

Then followed a period of bouts of headache and drowsiness, vomiting, and the treatment of a subgaleal fluid collection communicating with the underlying of cavity, which lasted for nine months after operation $\omega$ After six months, she had a Torkildsen-type ventriculer i cisternostomy from the cavity, using a rubber cathete⿻ and then a re-exploration of the cavity nine months after 을 hemispherectomy. The left ventricle was found to larger than it had originally been. The aqueduct was $\bar{C}$ obstructed, and was opened with a small catheter used a bougie. The lamina terminalis was opened with the ai $\Phi$ of providing more efficient cerebrospinal fluid circulatiog: $\vec{\theta}$ Lumbar puncture before this re-exploration had given $\frac{\pi}{9}$ clear colourless fluid with a protein level of $260 \mathrm{mg} /$ $100 \mathrm{ml}$.; the protein level never fell below $400 \mathrm{mg} . / 100 \mathrm{ml}$. during the three weeks after operation.

These early difficulties ended with the re-exploration. The next three years were good ones for her, and she attended a normal school with children of her own age. $\stackrel{\mathbb{Q}}{\Omega}$ Two years after hemispherectomy, she had a bout of $\underset{F}{\overrightarrow{2}}$ headache, vomiting, and drowsiness lasting for a day, $\frac{0}{3}$ with a few left-sided minor fits a few months afterwards. The left eye now tended to skew a little, but there was no other neurological change.

This three-year period ended in March 1958. A second bout of headache, vomiting, and drowsiness was now accompanied by pain in the back and legs. Lumbar:puncture revealed a heavily blood-stained spinal fluid with a xanthochromic supernatant layer, containing $2.8 \%$ haemoglobin, rising to $6.0 \%$ a few days later. Neurologically, there was now a left third nerve paresis and skew deviation of the eyeballs on lateral gaze. This attack $\frac{}{3}$ settled down and she was able to return to school. She $>$ had two further fairly good years. However, by July 1960 을 her mental shortcomings were becoming more apparent $\bar{\sim}$ and because of this she was excluded from school, and $O$ at this time, six years after operation, began to be ataxic. $N$ She deteriorated steadily from this point. The ataxia N worsened and was soon followed by nystagmus, dysarth- 
ria, and bouts of vomiting. By April 1961, she was unable to walk and was re-admitted. Lumbar puncture (unexpectedly, for she had no complaint of headache and no meningism) revealed a heavily blood-stained spinal fluid with a protein content of more than $1,000 \mathrm{mg} . / 100$ ml. By November 1961, the minor fits, left-sided as before, became rather more frequent. She was found to have gross cerebellar ataxia of the trunk, limbs, and head, bilateral sixth nerve paresis, and left-sided perceptive deafness. The posterior fossa was explored. The surface of the cerebellar hemispheres looked brown. The fourth ventricle was opened by dividing the vermis, and through it an enormously dilated aqueduct was seen. There was some improvement after operation, but the deterioration then re-established itself. On 11 December 1961, the posterior fossa was again explored. By this time, the pupils had become fixed and dilated, and the ocular movements were grossly disorganized. A left ventriculocisternostomy, using a polythene tube, was done, after a ventriculogram had shown an enormously dilated left ventricle. The opening into the fourth ventricle made two months previously had closed again. She never rallied after this operation and died four weeks afterwards, seven and a half years after hemispherectomy.

NECROPSY Post-mortem examination (R.I. P.M.No. 52/62) showed a wasted body, with obvious shortening of the left arm and leg. Sexual development was normal for the age. There was chronic cystitis, pyelonephritis, and

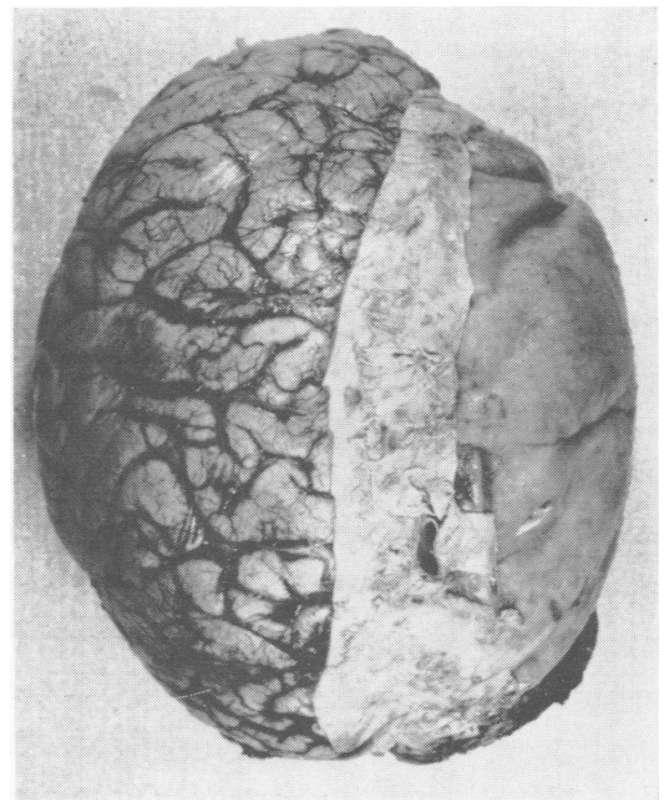

FIG. 2. Case 1. View of vertex, showing flattened convolutions on the left, and replacement of the right hemisphere by a m?mbranous sac. A midline strip of dura has been left. To the right of the cut edge of this, the tip of a rubber catheter is seen embedded in the membrane.

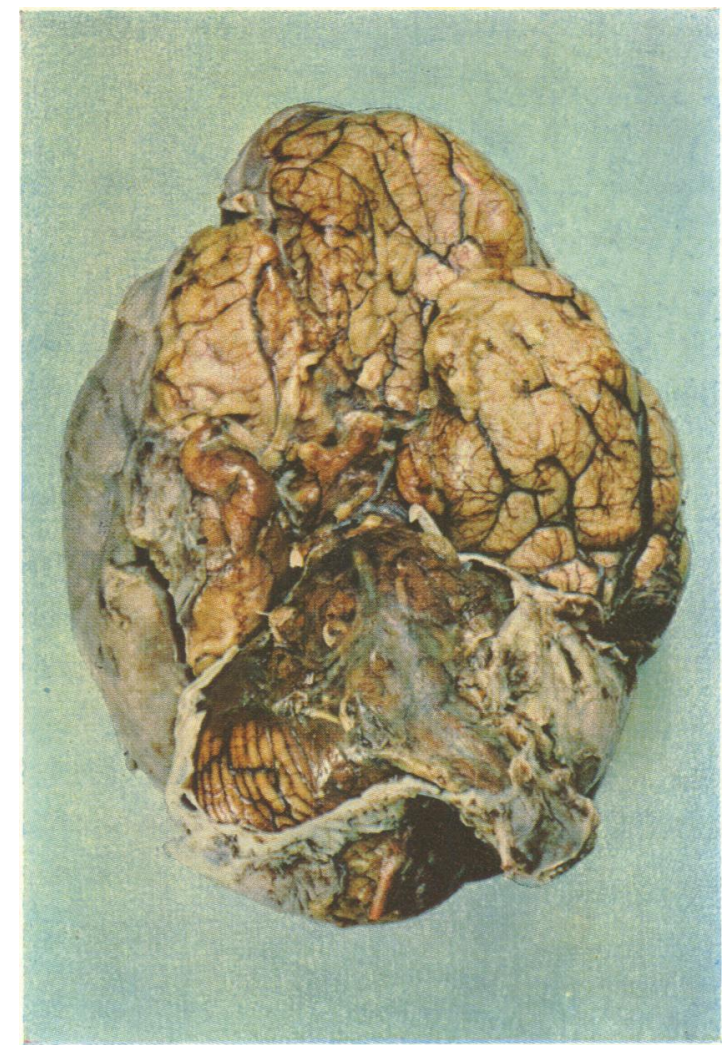

FIG. 3. Case 1. Base of brain, showing membrane and remnants of the right hemisphere. The meninges are brown and thickened. The lower end of the rubber catheter is embedded in fibrous tissue.

a terminal bronchopneumonia. The left common iliac vein contained thrombus, but there was no pulmonary embolism. Other significant findings were confined to the head.

Gross findings There was a marked asymmetry in the skull, the right cranial cavity being much smaller than the left. The bones of the vault were thinner than normal on the left and irregularly thickened on the right. There was a terminal thrombosis of the left lateral and superior petrosal sinuses, extending into the inferior anastomotic and several smaller veins. Beneath the dura on the right there was a tough membrane, about as thick as the dura itself, replacing the lost hemisphere and running on to the remains of the medial frontal and temporal cortex. At the vertex, embedded in fibrous tissue and lying between the dura and the membrane, was the rubber catheter which had been inserted eight years before (Fig. 2). The lower end of this catheter, also embedded in dense fibrous tissue, lay in the position of the cisterna magna. The recently inserted polythene tube was patent at both ends, the lower end lying in the subarachnoid space dorsal to the upper cervical cord, and the 


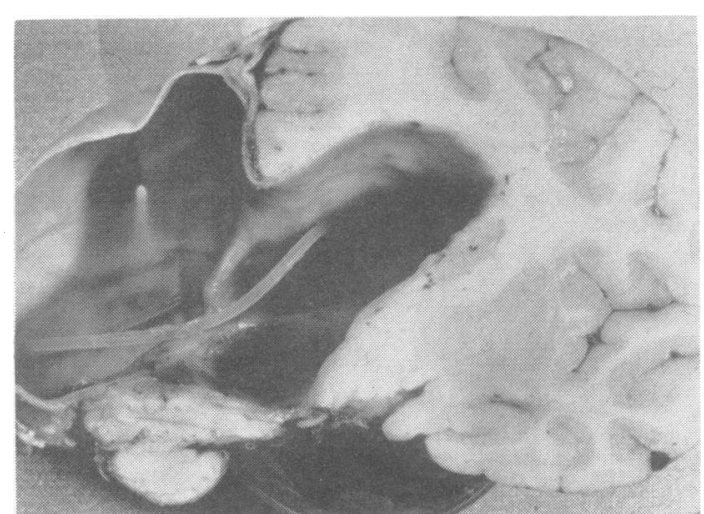

FIG. 4. Coronal section at the level of the hypothalamus, looking backwards. The ventricle is dilated; the corpus callosum and septum lucidum have vanished, and there is a common lining to the ventricle and the hemispherectomy cavity. The polythene tube comes from the posterior horn.

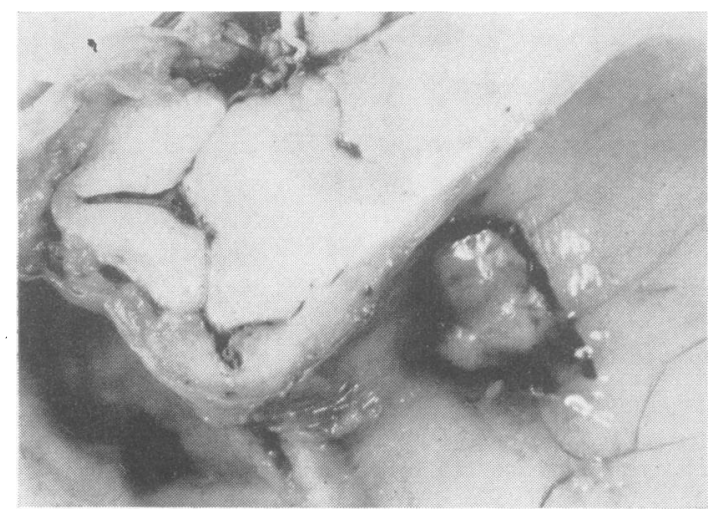

FIG. 5. Horizontal section through anterior horn showing the gelatinous grey lining of the ventricle, and an adherent lump of organized blood clot.

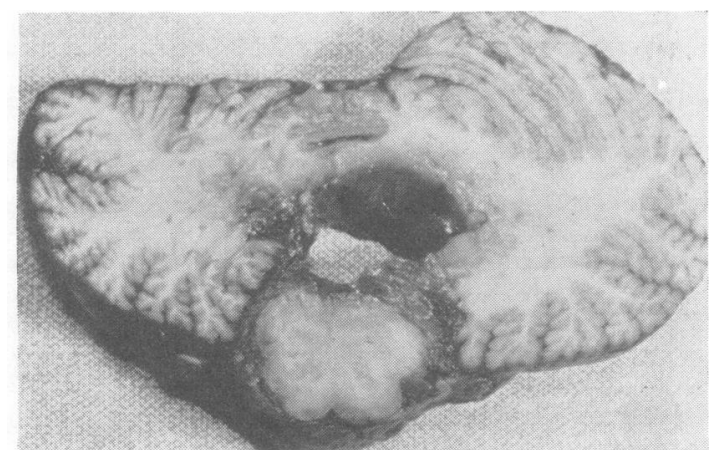

FIG. 6. Medulla and cerebellum at the level of the lateral recesses which are clogged with fibrous tissue and proliferated glia. The left cerebellar hemisphere is atrophic, and has undergone surgical damage. upper end entering the left lateral ventricle via an 으 occipital burr hole.

The convolutions of the left hemisphere showed severe hydrocephalic flattening. The whole of the base, including the optic chiasm, brain-stem, and cerebellum, and the spinal cord, showed deep brown staining and lepto- $C$ meningeal thickening (Fig. 3). The left cerebellar hemisphere was markedly atrophic.

Coronal slices through the cerebrum showed the cavity on the right side communicating through a broad opening into an extremely dilated ventricular system on the left. The third and lateral ventricles formed a single $\vec{F}$ cavity, as the septum and fornix had disappeared (Fig. 4). They were lined with a brownish membrane, $1-2 \mathrm{~mm}$. thick, glistening but with an irregular surface. This lining was continuous with the inner surface of the $\frac{\bar{s}}{5}$ cavity membrane. There was no massive accumulation $\mathbb{\otimes}$ of blood; but at a few points the ventricular lining showed small adherent plaques of recent blood clot (Fig. os 5). The remnants of the right hemisphere included the $\vec{\circ}$ gyrus rectus and medial orbital gyrus and the uncus of the hippocampal gyrus. There was hardly a trace of the right $\vec{\omega}$ thalamus and basal ganglia.

Sections of the brain-stem, cerebellum, and spinal cord showed superficial brown staining of nerve tissue $\bar{T}$ to a depth of up to $3 \mathrm{~mm}$. The right cerebral peduncle and pyramid were wasted, and particularly deeply stained $\dot{\omega}$ The aqueduct and fourth ventricle were enormous i dilated. The floor of the fourth ventricle was covered by $C$ thick layer of greyish-brown tissue, extending into this 을 lateral and posterior recesses, and blocking the foraming of Luschka and Magendie (Fig. 6). The superior part of $c$ the left cerebellar hemisphere showed severe atrophb and both dentate nuclei were discoloured and soft. The choroid plexus was shrivelled and rust-coloured. In te $\overrightarrow{0}$ cord, the most striking feature was the deep browi staining of the surface of the cord, contrasting with the almost normal colour of the nerve roots.

\section{HISTOLOGY ${ }^{1}$}

REMAINS OF RIGHT HEMISPHERE There were surviving $\stackrel{\otimes}{\mathbb{2}}$ nerve cells and myelinated fibres in the hippocampus, $\overrightarrow{\vec{P}}$ dentate fascia, hippocampal gyrus, putamen, and 웅 globus pallidus. Very few thalamic cells remained. The cingulate gyrus was reduced to a thin strip of glia. The temporal stump was covered on all surfaces by a fibrous layer continuous with the membrane.

LEFT HEMISPHERE There was a little haemosiderosis of the leptomeninges at the base, but not over the convexity. The cortex, hippocampus, striatum, pallidum, amygdaloid nucleus, and thalamus were 음 well preserved. The lateral geniculate body, though $\rightarrow$ heavily infiltrated with haemosiderin, did not appear to have lost cells. The white matter was grossly re- N

${ }^{1}$ This case, and the other two described in this paper, all showed $N$ superficial haemosiderosis of the central nervous system. We do not propose to describe this feature in detail, as it forms the subject of a $\omega$ further paper (Hughes and Oppenheimer, in preparation). 


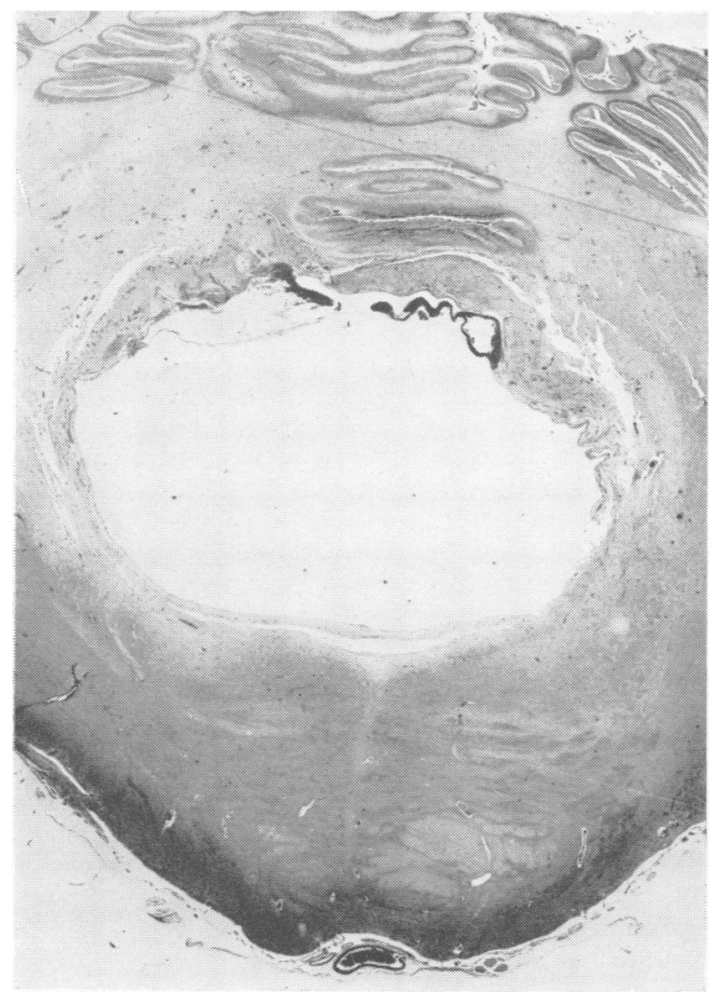

FIG. 7. Case 1. Section of pons, stained for iron, to show depth of subpial siderosis. Iron-laden phagocytes are also present in the walls of the dilated fourth ventricle.

duced in quantity, and the corpus callosum was totally degenerate.

VENTRICULAR SYSTEM The lining of the ventricles and aqueduct was abnormal throughout. Working from brain tissue towards the lumen, there was (a) a layer, rather thicker than normal, of subependymal glia; (b) a zone containing buried ependymal cells in clumps or canaliculi; (c) a layer of fibroglia, 1-2 mm. thick, containing thin-walled blood vessels, and scattered clusters of phagocytic cells laden with haemosiderin, often alongside vessels, with surrounding fibrous scarring; (d) over most of the surface of the lateral ventricle there was also a thin lining of collagenous tissue, continuous at its edges with the lining of the cavity membrane. The picture was in fact that of a severe so-called granular ependymitis, in which the 'granules' had coalesced into a continuous carpet of fibroglia, which had become vascularized, and in which multiple microscopic haemorrhages had occurred. The foramina of Luschka were blocked, partly by proliferated fibroglia, and partly by connective tissue, probably derived from the leptomeninges. The epithelium of exposed tufts of choroid plexus showed dense haemosiderosis, but there was no sign of bleeding from this source.

BRAIN-STEM AND SPINAL CORD There was total Wallerian degeneration of the right cerebral peduncle and pyramid; loss of tissue in the tectum and periaqueductal grey, due to gross dilatation of the aqueduct; and subpial haemosiderosis to a depth of 3 mm. (Fig. 7). All levels of the cord were affected. In striking contrast, the cranial and spinal nerve roots showed haemosiderosis only in their proximal, glia-containing, parts; beyond this, only occasional haemosiderin granules were seen. Haemosiderin was also present in the arachnoid granulations surrounding a spinal root ganglion.

CEREBELlum There was some disruption and scarring of the vermis, presumably dating from the operation in November 1961. There were also longstanding ischaemic changes in the superior twothirds of the left hemisphere. The foliar pattern was intact, but the whole tissue was shrunken; Purkinje and granule cells had disappeared, replaced by a layer of proliferated Bergmann glia cells. There was severe cell loss in the dentate nucleus. It was not clear whether these changes were a result of operation or of vascular insufficiency. On the right, there was fibre degeneration in the dentate hilum, without loss of cells in the nucleus. This was probably retrograde, due to distortion of the superior cerebellar peduncle. The exposed parts of the cerebellar cortex showed superficial haemosiderosis, with destruction of cortical nerve cells at the crowns of the folia. (This selective destruction, which is nearly always present in haemosiderosis of the central nervous system, indicates a peculiar susceptibility of cerebellar cortex to some breakdown product of blood.)

THE CAVITY MEMBRANE The membrane was about $\frac{1}{2} \mathrm{~mm}$. thick, and was composed of laminated fibrous tissue, sparsely cellular, with few blood vessels. Haemosiderin was present, not evenly scattered, but in rows of granules lying in particular laminae. This appearance suggested that during the period of formation of the membrane intermittent bleeding had occurred. Dense focal collections of pigment, suggestive of active bleeding points, such as were seen in the ventricular lining, were not present in the membrane except where it abutted on nervous tissue.

\section{CASE 2}

The birth of R.L. was uneventful. The early milestones were normal, and he was walking at 13 months. At this 
time, he had his first fit-a generalized convulsion without lateralizing features and for no obvious cause. He recovered quickly and completely from this, but two months later he had a febrile illness ushered in by a sudden access of unconsciousness and floppiness of all limbs lasting for five hours, succeeded by left-sided convulsions, which continued for several hours more. A left hemiplegia followed, which the parents described as 'just like a stroke'. He was admitted to a hospital with a diagnosis of polio-encephalitis and was treated in a respirator for several days. He stayed in hospital for four months. On discharge he had suffered a personality change in addition to the hemiplegia; he was much more aggressive, prone to temper tantrums and rages, unable to concentrate, and was less affectionate. Within three months, he was walking again with a hemiplegic gait. He did not begin to talk until the age of 4 , but then made good progress and became fully continent and started normal school. At age $4 \frac{1}{2}$ he began to have fits. At first the attacks lasted a few seconds only, with turning of the eyes to the left, and movements of the left face and arm. These occurred at the rate of one each week, but later they increased in frequency to six or seven daily. A year after they began he was excluded from school. The severity of the attacks worsened after three years, with falling, prolonged left-sided movements, and postictal sleep lasting one to two hours. Anticonvulsants had little influence on them. In 1952, aged 9, he had a welladapted left hemiplegic gait. He used the small spastic contracted left arm only for steadying objects. His behaviour was very aggressive and there were frequent rages. He was extremely distractable, his I.Q. being 67 on the Stanford-Binet scale. He could neither read nor write, but could feed himself efficiently.

On 12 March 1953 he had a right hemispherectomy. The excised tissue weighed $140 \mathrm{~g}$. There was very widespread cortical scarring, with thickened meninges. Destruction was most severe in the Rolandic, posterior parasagittal, and superior temporal areas. The underlying white matter was sclerotic. Histology showed all stages of cortical necrosis and ulegyria, but no vascular changes. The optic radiations were preserved, but the calcarine cortex was severely damaged.

Apart from one major fit on the first post-operative day, he had no more. The immediate deficit was a left homony- mous hemianopia and a transient loss of power in the으 left leg. There was some post-operative intracranial $Z$ bleeding, but red blood cells disappeared from the spinal fluid by the tenth day, although there was a slight re-O currence on the 14th day. The protein level in the spinal fluid on the eighteenth post-operative day was $320 \mathrm{mg}$. $100 \mathrm{ml}$. Apart from a pyrexia for two and a half weeks ${ }_{\infty}$ without other evidence of infection, the course was then straightforward. His parents were delighted by the result. $\overline{\bar{T}}$ His behaviour had improved markedly.

One year after operation, his left wrist was arthrodesed.. in order to make fuller use of the power in the left fingers, and the left tendo Achillis was lengthened. With home teaching, he learned to read and write, al-으 though neither very well. As he grew older, his intellec-흠 tual deficits became more obvious. In 1959, six years after $\overline{\bar{s}}$ operation, aged 16, he entered a sheltered workshop $\mathbb{\Phi}$ and did well there. However, he was still described as 'highly strung' and this aspect of the remaining behavioures disturbance was responsible for his dismissal from a job $\vec{\circ}$ in open manufacturing industry which he had held for $\rightarrow$ seven months. He never succeeded in finding another $\vec{\omega}$ job. He stayed at home for two years, but at age 20 . 10 years after operation, he was admitted to a residential centre for the handicapped. His steady deterioration begins from this time. In early 1963, he began to complain of headaches with pain in the back and legs, and $2 t$ ic the end of 1963 a tremor of the right limbs was observet His behaviour became worse, with rages, he becaneco incontinent, and later unable to walk. An E.E.G. showedo left-sided, generalized slow waves. In February $1964 \frac{2}{\circ}$ right-sided grasp reflex appeared, with weakness difde spasticity of the right limbs and right ankle clonus. The्ठुe was a left-sided perceptive deafness. A lumbar punctưfe at this time revealed yellow spinal fluid at normal pres sure, containing $2,000 \mathrm{mg} . / 100 \mathrm{ml}$. of protein. He was readmitted to the Radcliffe Infirmary. A lumbar air encephalogram showed air passing into the fourth ven-O tricle but not through the aqueduct, and externally into the pontine cisterns, but not through the cisterna ambiens. $\bar{O}$ He accordingly had an air ventriculogram (Fig. 8). The ڤొ ventricular fluid was yellow and contained a similar large amount of protein. There was marked dilatation of the third and left lateral ventricles, which communicated을

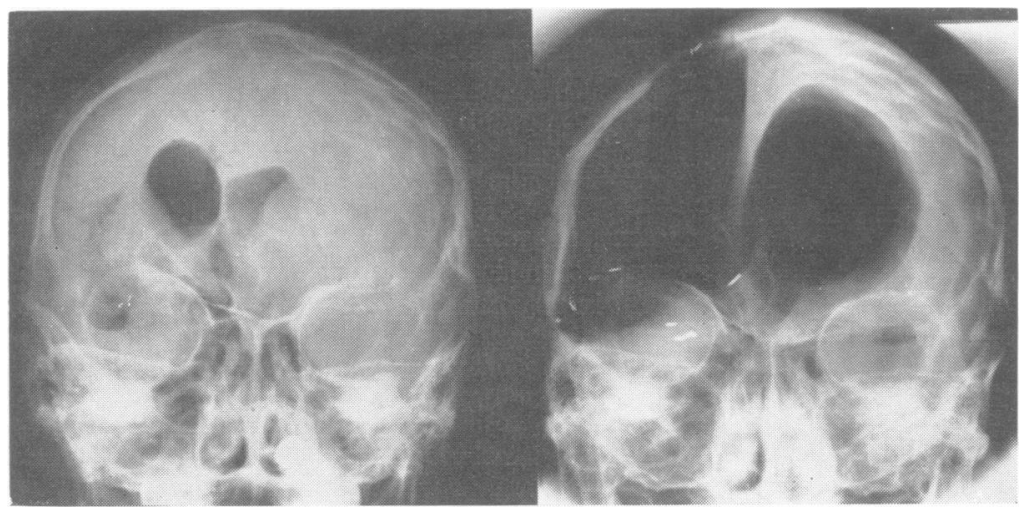

FIG. 8. Left: lumbar air encephalogram in 1952, showing a small right hemicranium, and thickened right calvarium, and atrophy of the right cerebral hemisphere. The left lateral ventricle is virtually normal.

Right: ventriculogram, February 1964, showing hydrocephalic dilatation of the previously normal left lateral ventricle. 


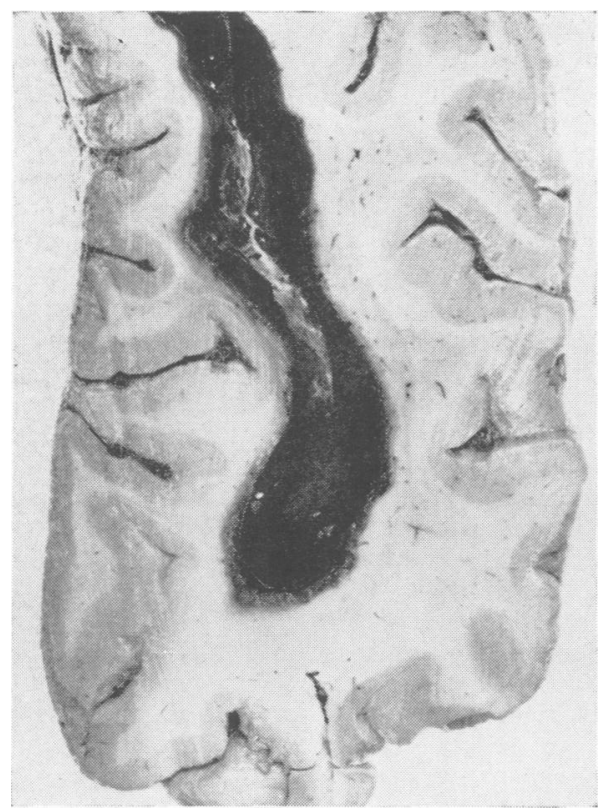

FIG. 9. Case 2. Posterior horn of left ventricle, looking backwards.

with the cavity. A block was demonstrated in the aqueduct, and confirmed by a Myodil ventriculogram. He underwent a Torkildsen-type ventriculocisternostomy from the left lateral ventricle. This operation did not alter his condition and he continued to deteriorate. He died two months after operation, 11 years after hemispherectomy.

NECROPSY The post-mortem findings were of a thin youth with left hemi-atrophy, and decubitus ulcers over the pelvis. The only other finding outside the head was of bilateral bronchopneumonia. The right side of the cranial cavity contained a large quantity of yellow fluid. A membrane, which was about as thick as the dura, was attached to the stumps of the most medial parts of the frontal and temporal lobes. The inner surface of the membrane was smooth and brown with occasional blackish plaques, suggestive of recent bleeding. The cavity led through a large opening into a somewhat distorted third ventricle, and thence by the left foramen of Monro to the lateral ventricle.

The left hemisphere showed hydrocephalic flattening, but no focal lesions. The body and anterior and posterior horns of the ventricle were dilated, and lined by a layer, 1-2 mm. thick, of brown or blackish tissue, with prominent vascular markings and numerous tiny excrescences (Fig. 9). This lining tissue was continuous, through the third ventricle, with the lining of the cavity membrane. Residual tissue on the right side included small parts of the cingulate and orbital cortex, hippocampus, uncus, and hippocampal gyrus, and a shrivelled thalamus and fornix.

The base, brain-stem, and cerebellum showed deep brown staining, extending about $2 \mathrm{~mm}$. inwards from the surface. The left cerebellar hemisphere was smaller than the right. The right cerebral peduncle and pyramid were wasted. The aqueduct was completely blocked by tough, greyish-brown tissue. The fourth ventricle was of normal shape and size, with some staining of its walls and the nodular thickenings of so-called granular ependymitis. The foramina of Luschka appeared to be patent. The choroid plexus was shrivelled and rust-coloured.

\section{HISTOLOGY}

REMAINS OF RIGHT HEMISPHERE There were persistent nerve cells and myelinated fibres in the hippocampus, dentate fascia, caudate nucleus, and globus pallidus. The putamen was absent. There were surviving cell groups in the thalamus, which probably represented the centrum medianum and intralaminar nuclei. The cingulate gyrus was reduced to a thin strip of glia, lying between layers of fibrous tissue derived from the cavity membrane. The temporal stump was likewise invested in layers of fibrous tissue, continuous with the membrane. The middle cerebral artery wall was degenerate, and the lumen showed organization with recanalization.

LEFT HEMISPHERE The leptomeninges at the base were thickened, and contained haemosiderin; elsewhere they looked normal. Cortex, hippocampus, striatum, pallidum and thalamus (including the lateral geniculate body) were well preserved. White matter was grossly reduced in amount. The corpus callosum was wholly degenerate.

VENTRICULAR SYSTEM The lining of the lateral and third ventricles closely resembled that in case 1 , with successive layers of subependymal glia, buried ependymal nests, and proliferated fibroglia. As before, this layer contained multiple collections of iron-laden phagocytes, associated with vessels, and with areas of fibrous scarring. Finally, there was a thin layer of collagenous tissue, continuous with the lining of the cavity membrane. The aqueduct was blocked, mainly by proliferated glia; in the centre of this was a small plug of connective tissue, containing iron-laden phagocytes, suggesting that the final occlusion was embolic, from above (Fig. 10). The line of ependymal cells suggested that at one time the aqueduct had been somewhat dilated. The fourth ventricle, in contrast to the other cases, was of normal shape and size, with patent exit foramina. There was a well-marked granular ependymitis, but the granulations had not coalesced, and there was some unburied ependyma. As in the previous case, the choroid plexus epithelium was heavily impregnated with haemosiderin. Haemosiderin was also seen at some places in the interstitial tissue. 


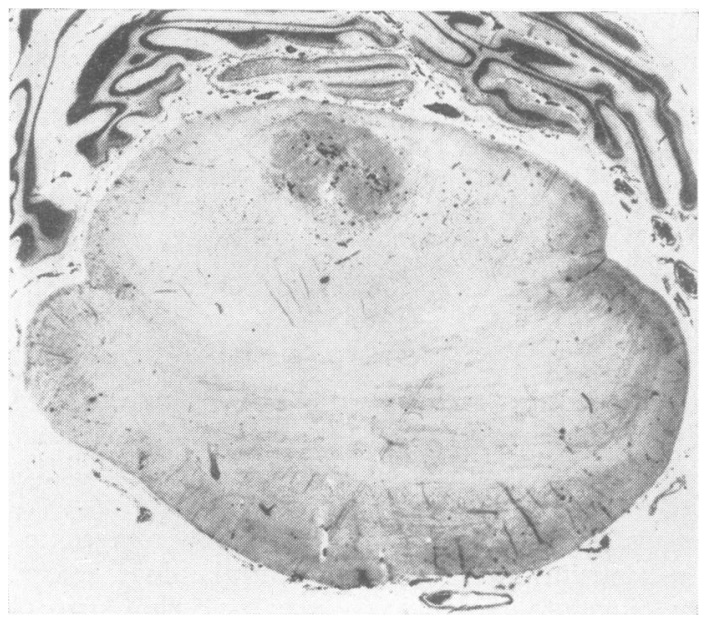

FIG. 10. Case 2. Section of upper pons, stained for iron, showing subpial siderosis and obstruction of the aqueduct by proliferated glia, containing iron-laden phagocytes.

BRAIN-STEM There was total degeneration of the right cerebral peduncle and pyramid, slight tissue loss in the peri-aqueductal grey, and subpial haemosiderosis to a depth of $1 \frac{1}{2} \mathrm{~mm}$.

CEREBELLUM The hila of both dentate nuclei, and the superior peduncles, showed some loss of myelinated fibres, probably retrograde. There was no obvious cell loss in the dentate nuclei. There was superficial haemosiderosis affecting the lower more than the upper surface, with local destruction of cortical cells; otherwise, the cortex and white matter were well preserved. No qualitative difference was seen between the two sides.

THE CAVITY MEMBRANE Most of the membrane, as in case 1 , consisted of sparsely cellular laminated fibrous tissue. In places, the membrane became thicker and divided into two layers, enclosing a more cellular granulation tissue, with dilated capillary spaces and abundant deposits of haemosiderin. These areas and the similar areas in the ventricular walls were considered to represent foci of previous haemorrhages.

\section{CASE 3}

The birth of D.H., a boy, was uneventful, but for many weeks there was a mild but lingering icterus neonatorum. Six weeks after birth the baby became limp and persistently drowsy. On examination, there were bruises over the trunk but no abnormal neurological signs. The fontanelle was tapped and a left subdural haematoma was found and drained through bifrontal burr holes. $\mathrm{He}$ was slow to thrive. At 9 weeks of age, a left lateral osteoplastic flap was turned, with the finding of a solid $\frac{\text { O }}{Z}$ blood clot in the Sylvian fissure, and damage to the $\mathbb{D}$ inferior surface of the frontal lobe. The clot and membrane were removed. Histologically the membrane, which of was over $1 \mathrm{~mm}$. thick, had the characteristic appearance of a subdural membrane, with layers of fibrous tissue enclosing pigment-laden phagocytes, dilated capillary spaces, and evidence of repeated extravasations. Subse- $\widehat{\underline{T}}$ quent progress was smooth, and he fed and gained weight normally. At 12 months he was noticed to have a mild right hemiparesis. At 13 months he had his first fit, which $:$ involved the right limbs, with turning of the head to the $\vec{F}$ right, going on to a major seizure. Phenobarbitone re- $-\vec{T}$ duced the severity of the attacks to episodes of sudden floppiness and sleepiness, occurring about twice weekly. $\frac{}{\bar{c}}$. His progress was slow; he could just walk at age 2 , and at $\frac{\sigma}{\sigma}$

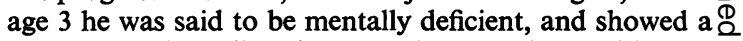
severe behaviour disturbance with aggression and hyperkinesis. By this time he could run about. At age 4, he could say only a very few words. The fits had decreased $\vec{O}$ in frequency to approximately six a year. When admitted $\overrightarrow{\mid}$ at 4 years and 8 months, he had a small head, a mild $\omega_{\sigma}$ spastic right hemiparesis and hemi-smallness, was aggressive, restless, destructive, dribbling, incontinent, and very backward in speech, with a mental age of about is 2 years. An electroencephalograph (E.E.G.) showed a left 0 parietal spike focus, with much theta activity. A ventro $\omega$ culogram demonstrated a dilated left ventricle comg $N$ municating with a large left frontal cyst. He had a le hemispherectomy on 25 September 1952, aged $5 \frac{1}{2}$. $\cong$ 음

The excised tissue weighed $180 \mathrm{~g}$. The frontal poite -

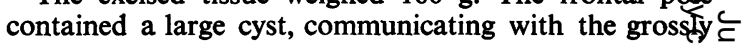
dilated lateral ventricle. The entire frontal cortex wogs shrunken and sclerotic, with thickened meninges. The $\vec{P}$ superior temporal and posterior Rolandic cortex wo also scarred, but the inferior temporal, posterior parietâto and occipital areas were well preserved. Histology showed the usual changes of ulegyria in the areas named, without demonstrating any underlying vascular lesion.

The immediate neurological deficit was a deepened right hemiparesis and a right homonymous hemianopia. $\frac{\circ}{\triangleright}$

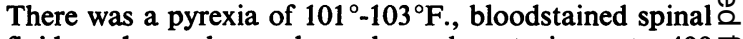
fluid, and a polymorphonuclear pleocytosis up to $400 \overrightarrow{\vec{\sigma}}$ cells/c.mm. for two weeks, with a raised spinal fluid 3 protein level, settling to $270 \mathrm{mg}$. $/ 100 \mathrm{ml}$. after four weeks. He was able to walk after this interval, and subsequent progress was satisfactory. Six months after operation, behaviour had improved and he had had no fits. Proximal movement had returned to the right arm, which could be lifted above the head, and he walked well. His speech, however, did not improve much, and the mental age never?. progressed beyond 3 years.

Three years after operation, he was able to live at home, went daily to an occupation centre, but was still incon-o tinent. Shortly after this period he began to have attacks of headache, vomiting, and photophobia. Although there $\frac{7}{0}$ were no overt fits until the terminal illness, he was now on occasions found lying limp in bed in the mornings. N This period of about one year passed and he improved once more, but at the end of 1960, eight and a half years $N$ after the operation, he began again to have daily vomiting $\mathbb{N}_{\mathcal{E}}$ and headache and was admitted after three months of $\bar{\sigma}$ 
this. Speech was rudimentary and dysarthric, there was difficulty in elevation of the right eye, and ankle clonus on both sides. Hearing was normal. A lumbar puncture gave yellow spinal fluid containing 4 white cells/c.mm. and $420 \mathrm{mg} . / 100 \mathrm{ml}$. protein. The operation cavity was tapped through a burr hole. It yielded lightly bloodstained brown fluid, containing 120 w.b.c./c.mm., 47,000 r.b.c./c. $\mathrm{mm}$., with a protein level of more than $1,000 \mathrm{mg} . / 100 \mathrm{ml}$. Air replacement was carried out, showing a dilated ventricular system, including the third ventricle and the aqueduct and fourth ventricle, but no air escaped into the cisterns. Two days later, his level of consciousness suddenly deteriorated and he went into uncontrolled status epilepticus with hyperpyrexia. Terminally, the cavity was tapped and found to be full of fresh blood.

NECROPSY Post-mortem examination (R.I. P.M. No. $103 / 61$ ) showed a rather thin child, with relative smallness of the right upper limb. There were no significant findings outside the head.

Gross findings The head was small, and the cranial cavity was smaller on the left than on the right. There was a well-healed bone flap on the left, and a recent left frontal burr-hole. The dura and the venous sinuses appeared normal.

On the left side, the cranial cavity above the tentorium was occupied by a tough fibrous membrane, thicker than the dura itself, and continuous at its margins with the shrivelled remains of the hemisphere. There were some fibrous adhesions between this membrane and the dura; for the rest, the two were separated by a massive fresh subdural haematoma. On the right side, there was a thin layer of fresh subdural blood, but no membrane.

The brain weighed $770 \mathrm{~g}$. The convolutions of the right cerebral hemisphere showed moderate hydrocephalic flattening, but no focal damage. The under surface, brain-stem, and cerebellum were all stained a deep brown, and the leptomeninges were thickened. The right cerebellar hemisphere was smaller than the left.

Coronal slices through the cerebrum showed a large cavity on the left, leading through a dilated foramen of Monro and a perforated septum lucidum to the third and right lateral ventricles. All parts of this cavity contained fresh blood clot, apparently originating in a number of large and small haemorrhages in the white matter surrounding the lateral ventricle, and unconnected with the fresh subdural clot, which extended between the falx and the membrane lining the cavity (Fig. 11).

The right lateral ventricle, third ventricle and aqueduct were grossly dilated. Instead of the normal ependymal lining, there was a layer, about $1 \mathrm{~mm}$. thick, of brownstained, roughened tissue, continuous on the left of the midline with the lining of the cavity membrane. The latter was firmly attached to the shrivelled remains of the corpus callosum, fornix, septum lucidum, thalamus, basal ganglia and medial temporal cortex. All of this residual tissue was stained a reddish brown.

Sections through the brain-stem and cerebellum showed superficial brown staining extending 2-3 mm. inwards. The abnormal lining of the third ventricle extended through a grossly dilated aqueduct to the fourth ventricle.

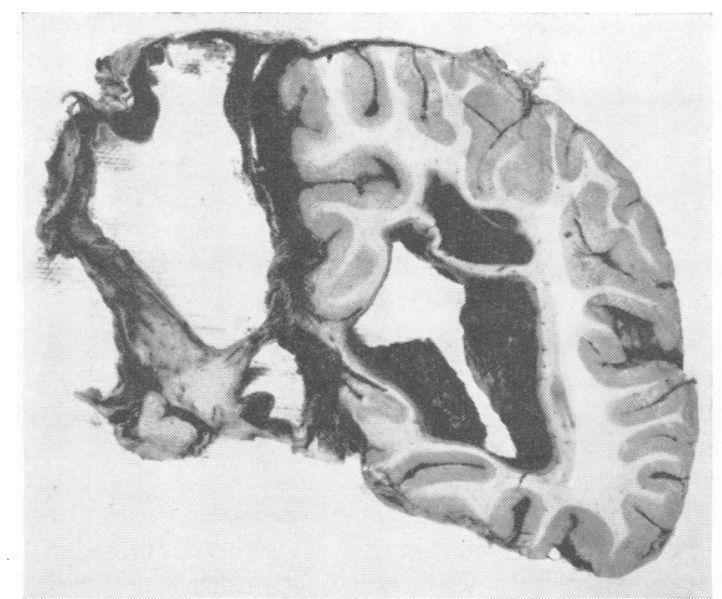

FIG. 11. Case 3. Coronal slice, showing multiple terminal haemorrhages (a) between the dura and the membrane, (b) into the common cavity of the membrane and the ventricle, and, (c) in the brain substance.

The foramina of Luschka and Magendie were blocked, partly by this lining tissue and partly by thickened leptomeninges. The choroid plexus of all the ventricles was stained brown. The left cerebral peduncle and pyramids were shrivelled and brown.

\section{HISTOLOGY}

REMAINS OF LEFT HEMISPHERE All the tissue was gliotic. The putamen and globus pallidus, though shrunken, were relatively preserved, with nerve cells and myelinated fibres. A few groups of thalamic cells remained. These were probably the centrum medianum and intralaminar nuclei, but they could not be clearly identified. Some pyramidal cells remained in the hippocampus, but the dentate fascia had disappeared. The cingulate gyrus was reduced to a thin strip of fibroglia, adherent to the membrane between it and the falx. All the surfaces of the cerebral stump were covered with a layer of fibrous connective tissue continuous with the cavity membrane. Some of the superficial arteries showed medial calcification.

RIGHT HEMISPHERE At the base, the leptomeninges were thickened, and contained a little intracellular iron. Over the convexities, they appeared normal. The cortex in all areas showed loss of cellslaminar, focal, or diffuse. The white matter was reduced in amount. The corpus callosum was totally degenerate and the anterior commisure greatly reduced in size. There was some loss of thalamic cells in the dorsomedial nucleus, and probably else- 
where. These changes were clearly of long standing, and could well be features of epileptic encephalopathy (Scholz, 1951). Another longstanding lesion, possibly associated with the original cerebral catastrophe, consisted in heavy crystalline deposits, containing iron and calcium, in the caudate nucleus, fornix, and stria terminalis. Finally, there were preterminal venous haemorrhages, of various sizes, scattered throughout the hemisphere.

VENTRICULAR SYSTEM As in case 1 , the ventricles and aqueduct were grossly dilated, and lined with a thick membrane composed of subependymal glia, buried ependymal nests, and a layer, 1-2 mm. thick, of fibroglia, containing thin-walled vessels and clusters of phagocytes laden with haemosiderin. Fibrous scarring was seen around these. The appearance was of a severe granular ependymitis, which had resulted in blockage of the outlets of the fourth ventricle. The ventricular system in this case differed from the other two in being covered internally with a layer of histiocytes and polymorphonuclears, indicative of a preterminal inflammatory reaction.

The choroid plexus of the lateral and fourth ventricles showed areas of degeneration and fibrosis. In the more exposed parts, the epithelial cells were heavily loaded with haemosiderin; but none was seen in the inner core of the plexus, and there was no sign that the plexus itself had been a source of bleeding.

BRAIN-STEM There was total Wallerian degeneration of the left cerebral peduncle, descending pontine bundles and pyramid, and tissue loss in the tectum and peri-aqueductal grey matter, clearly due to dilatation of the aqueduct. Subpial haemosiderosis, to a depth of $3 \mathrm{~mm}$., affected the whole brain-stem and as much of the cervical spinal cord as was available for study.

CEREBELLUM There was superficial haemosiderosis of the exposed folia on the under surface of both hemispheres, associated with destruction of cortical cells. For the rest, the cerebellar cortex, white matter and dentate nuclei appeared normal. There was no qualitative change to account for the relative smallness of the right hemisphere.

THE CAVITY MEMBRANE This was about $1 \mathrm{~mm}$. thick, and consisted of laminated fibrous tissue, with abundant blood vessels. There was fresh haemorrhage, not only on both surfaces (internal and juxtadural) but in the interstices of the fibrous laminae.

\section{DISCUSSION}

Clinically and anatomically, these three cases are strikingly similar. The essential features are:-

1 Infantile hemiplegia, treated in childhood by hemispherectomy.

2 A trouble-free period lasting for some years.

3 A period of deterioration, extending over several years, and ending in death. During this period there was evidence of bleeding into the cerebrospinal fluid pathways, and later of obstructive hydrocephalus.

4 Post-mortem findings of superficial haemosiderosis of the central nervous system: chronic granular ependymitis, leading to obstruction of cerebrospinal fluid pathways: and evidence of multiple bleeding points in the membrane which os had replaced the missing hemisphere, and in the $\vec{\circ}$ extension of this membrane onto the lining of the ventricular system.

Our task, then, is to attempt a reconstruction of the course of events leading to this final state of affairs. First, we must account for the haemosidero- i sis. This condition, first described by Noetzel (1940) extensively discussed by Rosenthal (1958) an $\bar{\Phi}$ i recently reviewed by Tomlinson and Walton (1964) 0 is now generally regarded as a consequence of the persistent or repeated presence of blood in thecerebrospinal fluid pathways. We may take it as $c$ reasonably certain that such bleeding had occurres in our patients. The questions to be answered cons $\vec{P}$ cern the cause of this bleeding, its source, and the time at which it occurred.

As to the last question, it is fairly certain that bleeding had occurred before the obstruction in the cerebrospinal fluid pathways became established. The evidence for this is the presence of iron pigment both in the lining of the ventricular system and in the $\mathbb{\perp}$ leptomeninges and subpial nervous tissue, and, in $\overrightarrow{\overrightarrow{0}}$ case 2 , on both sides of the aqueduct block. We 3 cannot be sure whether symptomless bleeding had $\bar{F}$ been going on during the apparently trouble-free $?$ period, or only at the end of this time. The lack of symptoms of meningism, even when an acute bleeding episode was known to be occurring, is note- $:$ worthy. We are also unable to say whether bleeding 3 continued after obstruction had occurred. In case 3 , there were terminal venous haemorrhages arising at $₹$ many points inside and outside the brain; but that 0 appears to have been a special kind of disaster, probably due to rapid changes of intracranial pressure following ventriculography.

As to the source of the bleeding, no single site $N$ can be incriminated. There is no sign that bleeding $N$ occurred from any of the larger arteries and veins $N$ divided at operation; nor of focal haemorrhagic 
lesions in the nervous tissue or choroid plexus. On the other hand we have the histology of the cavity membrane and of the ventricular lining, which points in each case to the existence of multiple bleeding points in the innermost layers of these structures. We believe, in fact, that bleeding occurred at many points and at different times, and that the blood in the cavity and ventricles found its way through the exit foramina to the subarachnoid space.

In addition to superficial haemosiderosis, another effect attributable to repeated bleeding is the universal granular ependymitis, and it was this which finally resulted in obstruction. Proliferation of the subependymal glia, with the formation of granular projections into the ventricular cavity, and 'burying' of groups of ependymal cells, is a well-known phenomenon, which appears to be a non-specific reaction to irritation. In our three cases the obvious irritant is blood, or some product of its breakdown.

We cannot be at all certain as to the cause of the bleeding, but we believe that there is a clue in the nature of the cavity membrane. Histologically this is indistinguishable from the membrane of a chronic subdural haematoma; it has the same irregular lamination of fibrous tissue, with clefts containing inflammatory cells and blood pigments, and a similar bloodstained lining of granulation tissue. The cavity membrane is, in fact, a subdural membrane, differing from the membrane of a chronic haematoma by being continuous at its edges with brain tissue, and in its cavity being continuous with the ventricles. In this connexion, it would be interesting to know the state of the brain and the membranes in the more fortunate 14 patients. Presumably a membrane always forms, separating the dura from the hemispherectomy cavity. We would predict that this membrane, in non-haemorrhagic cases, would be relatively tenuous and avascular, and similar to that found in cases of resolved subdural hygroma. We have no solid reason to think that any of our cases suffered from a bleeding tendency, although this had been considered in case 3 at the time of his initial cerebral haemorrhage, in view of the prolonged jaundice.

For various reasons it is difficult at this stage to disentangle individual clinical features of the period of decline and to ascribe them either to the hydrocephalus on the one hand or to the haemosiderosis on the other. According to Tomlinson and Walton (1964), the most constant clinical features of haemosiderosis are nerve deafness, dementia, and ataxia. In our cases, two were known finally to have mild nerve deafness, and the hearing of the third seemed normal. In addition, all three had an undoubted mental deterioration; all eventually were ataxic and had a tremor of the previously unaffected limbs, features met with commonly in severe acquired hydrocephalus.

Following hemispherectomy, the ventricular system and the surface of the brain and cord were exposed to a highly abnormal proteinaceous ironrich fluid, vastly different from cerebrospinal fluid, with an eventually fatal result. It would not be out of place to suggest ways both of avoiding this difficulty, and of meeting it once it has occurred. In order to avoid connecting the cerebral ventricles to a large sump lined by vascular connective tissue, which is in effect the result of the present standard operation, it may be possible to devise a technique for functionally disconnecting the abnormal hemisphere from the rest of the brain, leaving it in place, with an intact ventricular lining. When the affected hemisphere is shrunken and sclerotic this might be technically difficult, and we do not know with any certainty which connexions are the important ones. Another possible solution would involve, after removing the hemisphere, an attempt to close off the cavity from the ventricles, taking up the space in the cavity with a biologically inert prosthesis.

Once repeated bleeding was recognized to have taken place, the membrane could be excised. We know that this has been done, apparently with some success (Falconer, 1965, personal communication). This might have the effect of making the situation worse, in that stripping an adherent vascular membrane from vascular dura would tend to promote further bleeding. This manoeuvre, too, would deal only with a part of the abnormal lining, the ventricular wall being inaccessible and, judging by its appearance in our cases, just as likely to bleed as the cavity membrane. However, as the outcome otherwise seems to be disastrous, excision of the membrane would be the best procedure. In two of our cases ventriculocisternostomy was ineffective in dealing with the obstructive hydrocephalus, and third ventriculostomy or a ventriculo-atrial shunt might be more effective.

The operation of hemispherectomy has, we think, declined in popularity. Yet follow-up study of the surviving cases from this centre has convinced us that in a proportion of cases of infantile hemiplegia hemispherectomy is a highly worthwhile procedure; and for this reason we think it is worth making an effort to improve the standard operative technique, in order to avoid complications such as the one we have been describing.

\section{SUMMARY}

Clinical and anatomical details are given of three patients dying of a late complication of the operation of hemispherectomy for infantile hemiplegia. The 
cause of death in each case appears to have been persistent bleeding into the cerebrospinal fluid pathways, causing granular ependymitis, obstructive hydrocephalus, and superficial haemosiderosis. Suggestions are made of ways in which this complication might be avoided.

Our thanks are due to Mr. Joe Pennybacker whose patients these were and who has provided us with encouragement, advice, and criticism, and to Mr. Walpole Lewin, who operated upon case 2. The drawing of the operation was provided by Miss Audrey Arnott.

\section{REFERENCES}

Dandy, W. E. (1928). Removal of right cerebral hemisphere for certain tumors with hemiplegia. J. Amer. med. Ass., 90, 823-825.

Krynauw, R. A. (1950). Infantile hemiplegia treated by removing one $\mathcal{C}$ cerebral hemisphere. J. Neurol. Neurosurg. Psychiat., 13,
243-267.

Noetzel, H. (1940). Diffusion von Blutfarbstoff in der inneren Rand- $\square$ zone und äusseren Oberfläche des Zentralnervensystems bei $\mathscr{C N}$ subarachnoidaler Blutung. Arch. Psychiat. Nervenkr., 111, $\Omega$ 129-138.

Rosenthal, P. (1958). Siderose der Randzonen des Zentralnervensystems. Dtsch. Z. Nervenheilk., 178, 431-472.

Scholz,W. (1951). Die Krampfschädigungen des Gehirns. Springer, Berlin.

Tomlinson, B. E., and Walton, J. N. (1964). Superficial haemosiderosis of the central nervous system. J. Neurol. Neurosurg. Psychiat., 27, 332-339. 